\title{
Axion Constraints from Quiescent Soft Gamma-ray Emission from Magnetars
}

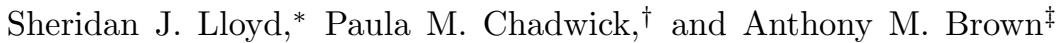 \\ Centre for Advanced Instrumentation, Dept. of Physics, \\ University of Durham, South Road, Durham, DH1 3LE, UK \\ Huai-Ke Gud $\$$ and Kuver Sinha \\ Department of Physics and Astronomy, University of Oklahoma, Norman, OK 73019, USA
}

(Dated: December 15, 2020)

\begin{abstract}
Axion-like-particles (ALPs) emitted from the core of a magnetar can convert to photons in its magnetosphere. The resulting photon flux is sensitive to the product of $(i)$ the ALP-nucleon coupling $G_{a n}$ which controls the production cross section in the core and $(i i)$ the ALP-photon coupling $g_{a \gamma \gamma}$ which controls the conversion in the magnetosphere. We study such emissions in the soft-gammaray range $(300 \mathrm{keV}$ to $1 \mathrm{MeV})$, where the ALP spectrum peaks and astrophysical backgrounds from resonant Compton upscattering are expected to be suppressed. Using published quiescent softgamma-ray flux upper limits in 5 magnetars obtained with CGRO COMPTEL and INTEGRAL SPI/IBIS/ISGRI, we put limits on the product of the ALP-nucleon and ALP-photon couplings. We also provide a detailed study of the dependence of our results on the magnetar core temperature. We further show projections of our result for future Fermi-GBM observations. Our results motivate a program of studying quiescent soft-gamma-ray emission from magnetars with the Fermi-GBM.
\end{abstract}

\section{INTRODUCTION}

The axion arises as a solution to the strong CP problem of QCD and is a plausible cold dark matter candidate 115]. The search for axions, and more generally axionlike-particles (ALPs) (for which the relationship between particle mass and the Peccei-Quinn scale is relaxed), now spans a vast ecosystem including helioscopes, haloscopes, interferometers, beam dumps, fixed target experiments, and colliders 6 .

This paper concerns indirect detection of ALPs, specifically their conversion into photons in the magnetospheres of neutron stars with strong magnetic fields (magnetars) 7 9]. The mechanism is as follows 1 . relativistic ALPs $(a)$ emitted from the core by nucleon $(N)$ bremsstrahlung (from the Lagrangian term $\left.\mathcal{L} \supset G_{a n}\left(\partial_{\mu} a\right) \bar{N} \gamma^{\mu} \gamma_{5} N\right)$ escape into the magnetosphere, where they convert to photons (from the Lagrangian term $\left.\mathcal{L} \supset-\frac{1}{4} g_{a \gamma \gamma} a F_{\mu \nu} \tilde{F}^{\mu \nu}\right)$ in the presence of the neutron star magnetic field $B$. The ALP emission rate strongly depends on the core temperature, $T_{c}$, as $T_{c}^{6} 112$ and

\footnotetext{
* sheridan.j.lloyd@durham.ac.uk

$\dagger$ p.m.chadwick@durham.ac.uk

$\ddagger$ anthony.brown@durham.ac.uk

$\S$ ghk@ou.edu

ศ kuver.sinha@ou.edu

1 The conversion of relativistic ALPs near neutron stars begins with [10] where the probability of conversion was overestimated, followed by the classic paper [11] which correctly accounted for non-linear QED and the photon mass in the ALP-photon conversion equations. In [11] an order of magnitude calculation of the conversion probability near the magnetar surface concluded that it was too small to produce observable signals (the photon mass term dominates over the ALP-photon mixing term at the surface). However, the conversion becomes appreciable away from the surface, due to the different scaling of the photon mass $\left(\sim 1 / r^{6}\right)$ compared to the ALP-photon mixing $\left(\sim 1 / r^{3}\right)$.
}

13 while the conversion rate generally increases with stronger $B$, making magnetars, with their high $T_{c} \sim 10^{9}$ $\mathrm{K}$ and strong $B \sim 10^{14} \mathrm{G}$, a natural target for these studies.

The purpose of this paper is to initiate an investigation of the signals resulting from ALP-photon conversions in the quiescent soft-gamma-ray spectrum $(300 \mathrm{keV}-1$ $\mathrm{MeV}$ ) from magnetars, similar to probes in the X-ray band in magnetars $[7$ and 8 and in pulsars [14. Since the peak of photon energies arising from ALP-photon conversion lies in the soft-gamma-ray band, this is an especially important regime to explore. Moreover, while searches for new physics in the soft and hard X-ray emission from magnetars must contend with background from thermal emission and resonant Compton upscattering respectively, the astrophysical background in the softgamma-ray regime is relatively suppressed as we discuss in SecVII

Starting with the photon polarization tensor, we provide in SecVI expressions for the photon refractive indices in the strong and weak magnetic field regimes for photon energies $\omega \lesssim 2 m_{e}$, where $m_{e}$ is the electron mas $\AA^{2}$ In Sec III, the coupled ALP-photon propagation equations are then solved numerically using the appropriate refractive indices. In Sec IV, the production in the magnetar core is discussed: this proceeds via bremsstrahlung from neutrons $\psi_{n}: \psi_{n}+\psi_{n} \leftrightarrow \psi_{n}+$ $\psi_{n}+a$. Combining all of the above ultimately yields the photon luminosity coming from ALP-photon conversions $L_{a \rightarrow \gamma}$, as well as the spectral energy distribution. These quantities are obtained for a selection of 5 magnetars: 1E 2259+586, 4U 0142+61, 1E 1048.1-5937,

\footnotetext{
${ }^{2}$ In the soft-gamma-ray regime, one has to start directly from the photon polarization tensor and take appropriate limits, instead of starting with the Euler-Heisenberg Lagrangian.
} 
1RXS J170849.0-400910 and 1E 1841-045. Using published quiescent soft-gamma-ray flux upper limits (ULs), constraints are then put on the product of couplings $G_{a n} \times g_{a \gamma \gamma}$ using a spectral analysis whose details are shown in Sec $\mathrm{X}$

The main message of our paper is that quiescent softgamma-ray emission from magnetars is a fertile target to investigate the physics of ALPs. The Fermi-GBM is a very useful instrument to determine the UL soft-gammaray fluxes of the 23 confirmed magnetars and such a study could yield very restrictive constraints on $G_{a n} \times g_{a \gamma \gamma}$.

\section{PHENOMENOLOGY}

In this section, we discuss the predicted luminosity from ALP-photon conversion in the magnetosphere. We assume a dipolar magnetic field defined by

$$
B=B_{\text {surf }}\left(\frac{r_{0}}{r}\right)^{3} \text {. }
$$

ALPs propagating radially outwards from a magnetar obey the following evolution equations derived in [11]

$$
\begin{gathered}
i \frac{d}{d x}\left(\begin{array}{c}
a \\
E_{\|} \\
E_{\perp}
\end{array}\right)=\left(\begin{array}{ccc}
\omega r_{0}+\Delta_{a} r_{0} & \Delta_{M} r_{0} & 0 \\
\Delta_{M} r_{0} & \omega r_{0}+\Delta_{\|} r_{0} & 0 \\
0 & 0 & \omega r_{0}+\Delta_{\perp} r_{0}
\end{array}\right)\left(\begin{array}{c}
a \\
E_{\|} \\
E_{\perp}
\end{array}\right), \quad \text { where } \\
\Delta_{a}=-\frac{m_{a}^{2}}{2 \omega}, \quad \Delta_{\|}=\left(n_{\|}-1\right) \omega, \quad \Delta_{\perp}=\left(n_{\perp}-1\right) \omega, \quad \Delta_{M}=\frac{1}{2} g_{a \gamma \gamma} B \sin \theta .
\end{gathered}
$$

The parallel and perpendicular electric fields are denoted by $E_{\|}(x)$ and $E_{\perp}(x)$, respectively, while $a(x)$ denotes the ALP field. The distance from the magnetar is given by the rescaled dimensionless parameter $x=r / r_{0}$, where $r$ is the distance from the magnetar and $r_{0}$ its radius. The energy of the photon is given by $\omega$, the ALP mass by $m_{a}$, and the ALP-photon coupling by $g_{a \gamma \gamma} . \theta$ is the angle between the direction of propagation and the $B$ field.

The refractive indices $n_{\|}$and $n_{\perp}$ are obtained from the photon polarization tensor, which can be worked out at one-loop level in various limits of the photon energy $\omega$ and the strength of the magnetic field $B$ relative to the quantum critical magnetic field $B_{c}$, given by $B_{c}=$ $m_{e}^{2} / e=4.413 \times 10^{13} \mathrm{G}$. Here $e=\sqrt{4 \pi \alpha}$ and the fine structure constant $\alpha \approx 1 / 137$.

Near the surface, the $B$-field of the magnetars we consider typically exceeds $B_{c}$, so that $\omega \lesssim 2 m_{e}$ and $B>B_{c}$. The corresponding refractive indices are given in Eq. C15. Given the spatial dependence from Eq. 1, the magnetic field decreases to below the critical strength at a distance $\sim 3 r_{0}$. Beyond that, we are in a regime where $\omega \lesssim 2 m_{e}$ and $B \ll B_{c}$, with $\left(\frac{\omega}{2 m_{e}}\right)^{2}\left(\frac{B}{B_{c}}\right)^{2} \ll 1$. The corresponding refractive indices are given in Eq. 43 . For further details, see Sec VI.

After calculating the parallel refractive index $n_{\|}$, the probability of conversion can be obtained as a function of $g_{a \gamma \gamma}$ and the mass $m_{a}$ by numerically solving Eq. 2 . The interesting regime for conversion is $r=r_{a \rightarrow \gamma} \sim$ $\mathcal{O}(1000) r_{0}$ (the "radius of conversion"), where the conversion probability becomes large. This arises from the
ALP-photon mixing becoming maximal when $\Delta_{M} \sim \Delta_{\|}$. Far away from the surface, $\Delta_{M} \sim 1 / r^{3}$, while $\Delta_{\|} \sim 1 / r^{6}$ from Eq. 43 , with the two becoming equal around $r_{a \rightarrow \gamma}$.

Along with the probability of conversion, we require the normalized ALP spectrum and the number of ALPs being produced from the magnetar core. Integrating the product of these quantities over the ALP energy range $\omega \subset\left(\omega_{i}, \omega_{f}\right)=(300 \mathrm{keV}, 1000 \mathrm{keV})$ gives us the final predicted luminosity from ALP-photon conversions. Our master equations for the final predicted theory photon luminosity are Eq. 12 - Eq. 17, which we solve numerically. A semi-analytic calculation following [8] is also performed to validate our results. We provide further details in Sec III and Sec IV

\section{ALP-PHOTON PROBABILITY OF CONVERSION}

In this section, we provide details of the propagation of the ALP-photon system through the magnetosphere, with the aim of deriving the probability of conversion $P_{a \rightarrow \gamma}(\omega, \theta)$. Our treatment largely follows the framework developed by one of the authors in [7, 8. For later work that followed these initial calculations, we refer to [14]. We note that 21] performed detailed numerical computations of the conversion probability in the soft X-ray thermal emission band, and our results agree with theirs in the appropriate limit. We note in passing that ALP decays can be neglected.

The propagation of the system is governed by Eq. 2 and Eq. 3. while the relevant refractive indices will be 


\begin{tabular}{|c|c|c|c|c|}
\hline Magnetar & $\begin{array}{c}\text { Distance } \\
\mathrm{kpc}\end{array}$ & $\begin{array}{l}\text { Surface } B \\
\text { Field } \\
10^{14} \mathrm{G}\end{array}$ & $\begin{array}{l}\text { Age } \\
\text { kyr }\end{array}$ & $\begin{array}{c}\text { UL Flux } \\
300 \mathrm{keV}-1 \mathrm{MeV} \\
10^{-10} \mathrm{erg} \mathrm{cm}^{-2} \mathrm{~s}^{-1}\end{array}$ \\
\hline $1 \mathrm{E} 2259+586$ & $3.2_{-0.2}^{+0.2[15}$ & 0.59 & 230 & $1.17[16$ \\
\hline $4 \mathrm{U} 0142+61$ & $3.6_{-0.4}^{+0.4} 17$ & 1.3 & 68 & $8 . 1 6 \longdiv { 1 8 }$ \\
\hline 1RXS J170849.0-400910 & $3.8_{-0.5}^{+0.5} 15$ & 4.7 & 9 & 1.9216 \\
\hline $1 \mathrm{E} 1841-045$ & $8.5_{-1.0}^{+1.3} 19$ & 7 & 4.6 & $2 . 5 6 \longdiv { 1 6 }$ \\
\hline 1E 1048.1-5937 & $9.0_{-1.7}^{+1.7} 17$ & 3.9 & 4.5 & $3.04[16$ \\
\hline
\end{tabular}

TABLE I. Magnetar sample with sum of UL flux in the $300 \mathrm{keV}-1 \mathrm{MeV}$ band. UL fluxes and distances are from the references shown, surface $B$ field and age are from the online ${ }^{27}$ version of the McGill magnetar catalog [20].

presented in Sec VI It is clear from the structure of the mixing matrix in Eq. 2 that $E_{\perp}$ does not mix with the ALP; we will thus not consider it any further. It is convenient to reparametrize the other fields as follows:

$$
\begin{aligned}
a(x) & =\cos [\chi(x)] e^{-i \phi_{a}(x)}, \\
E_{\|}(x) & =i \sin [\chi(x)] e^{-i \phi_{E}(x)},
\end{aligned}
$$

where $\chi(x), \phi_{a}(x)$ and $\phi_{E}(x)$ real functions. The propagation equations then simplify to

$$
\begin{aligned}
\frac{d \chi(x)}{d x} & =-D(x) \cos [\Delta \phi(x)], \\
\frac{d \Delta \phi(x)}{d x} & =A(x)-B(x)+2 D(x) \cot [2 \chi(x)] \sin [\Delta \phi(x)],
\end{aligned}
$$

where $\chi(1)$ is the initial state at the surface of the magnetar, and we have defined the relative phase $\Delta \phi(x)=$ $\phi_{a}(x)-\phi_{E}(x)$. For pure initial states, the initial condition for $\Delta \phi(1)$ satisfies $\Delta \phi(1)=m \pi$ with $m \in \mathbb{Z}$. For a pure ALP initial state it is therefore possible to set $\Delta \phi(1)=0$. The ALP-photon conversion probability is then simply

$$
P_{a \rightarrow \gamma}(x)=\sin ^{2}[\chi(x)] .
$$

Our results for the conversion probability will be based on a full numerical solution to the evolution equations. The probability of conversion $P_{a \rightarrow \gamma}$ is thus obtained by numerically solving the propagation equations in Eq. 2 . For the calculations, we need the refractive indices that appear in Eq. 3. These refractive indices are derived in Sec VI

We now outline a semi-analytic solution that agrees very well with our full numerical solution. The semianalytic solution can be obtained by analogy with timedependent perturbation theory in quantum mechanics, leading to 11

$$
\begin{aligned}
& P_{a \rightarrow \gamma}(x)=\mid \int_{1}^{x} d x^{\prime} \Delta_{M}\left(x^{\prime}\right) r_{0} \\
& \quad \times\left.\exp \left\{i \int_{1}^{x^{\prime}} d x^{\prime \prime}\left[\Delta_{a}-\Delta_{\|}\left(x^{\prime \prime}\right)\right] r_{0}\right\}\right|^{2} \\
& =\left(\Delta_{M 0} r_{0}\right)^{2}\left|\int_{1}^{x} d x^{\prime} \frac{1}{x^{\prime 3}} \exp \left[i \Delta_{a} r_{0}\left(x^{\prime}-\frac{x_{a \rightarrow \gamma}^{6}}{5 x^{\prime 5}}\right)\right]\right|^{2} .
\end{aligned}
$$

These equations are accurate for small enough values of $g$, which fall in the regime we are interested in. The second expression utilized the dimensionless conversion radius, where the probability of conversion becomes maximal

$$
x_{a \rightarrow \gamma}=\frac{r_{a \rightarrow \gamma}}{r_{0}}=\left(\frac{7 \alpha}{45 \pi}\right)^{1 / 6}\left(\frac{\omega}{m_{a}} \frac{B_{0}}{B_{c}}|\sin \theta|\right)^{1 / 3} .
$$

This is valid when the conversion radius is much larger than the radius of the magnetar. In that limit $\hat{q}_{\|} \rightarrow 1$ and the integral in the exponential can be trivially calculated. The conversion probability becomes

$$
\begin{aligned}
P_{a \rightarrow \gamma}(x)=\left(\frac{\Delta_{M 0} r_{0}^{3}}{r_{a \rightarrow \gamma}^{2}}\right)^{2} \mid \int_{\frac{r_{0}}{r_{a \rightarrow \gamma}}}^{\infty} d t \frac{1}{t^{3}} \\
\quad \times\left.\exp \left[i \Delta_{a} r_{a \rightarrow \gamma}\left(t-\frac{1}{5 t^{5}}\right)\right]\right|^{2},
\end{aligned}
$$

where the norm of the integral in $(9)$ is order one for our benchmark points. We can further simplify the expression in the large $\left|\Delta_{a} r_{a \rightarrow \gamma}\right|$ regime by using the method of steepest descent, and the small $\left|\Delta_{a} r_{a \rightarrow \gamma}\right|$ regime with a change of variables:

$$
\begin{aligned}
P_{a \rightarrow \gamma}(x) & =\left(\frac{\Delta_{M 0} r_{0}^{3}}{r_{a \rightarrow \gamma}^{2}}\right)^{2} \\
& \times\left\{\begin{array}{ll}
\frac{\pi}{3\left|\Delta_{a} r_{a \rightarrow \gamma}\right|} e^{\frac{6 \Delta_{a} r_{a \rightarrow \gamma}}{5}} & \left|\Delta_{a} r_{a \rightarrow \gamma}\right| \gtrsim 0.45 \\
\frac{\Gamma\left(\frac{2}{5}\right)^{2}}{5^{\frac{6}{5}}\left|\Delta_{a} r_{a \rightarrow \gamma}\right|^{\frac{4}{5}}} & \left|\Delta_{a} r_{a \rightarrow \gamma}\right| \lesssim 0.45
\end{array} .\right.
\end{aligned}
$$

We display the function $\chi(x)$ and the probability of conversion as a function of the radial distance in Fig. 1 . These plots are obtained from a full numerical solution to the evolution equations

Before closing this section, we also provide an heuristic way of studying the conversion probability. The mixing angle between $E_{\|}(x)$ and the ALP $a(x)$ is given by

$$
\tan 2 \theta_{\text {mix }}=\frac{\Delta_{M}}{\left(\Delta_{a}-\Delta_{\|}\right) / 2} \sim \frac{g B}{\left(1-n_{\|}\right) \omega} .
$$

For benchmark values of the magnetic field and other parameters relevant for this work, one can check that at the surface of the magnetar the mixing is negligible. However, the mixing (and hence the probability of conversion) 

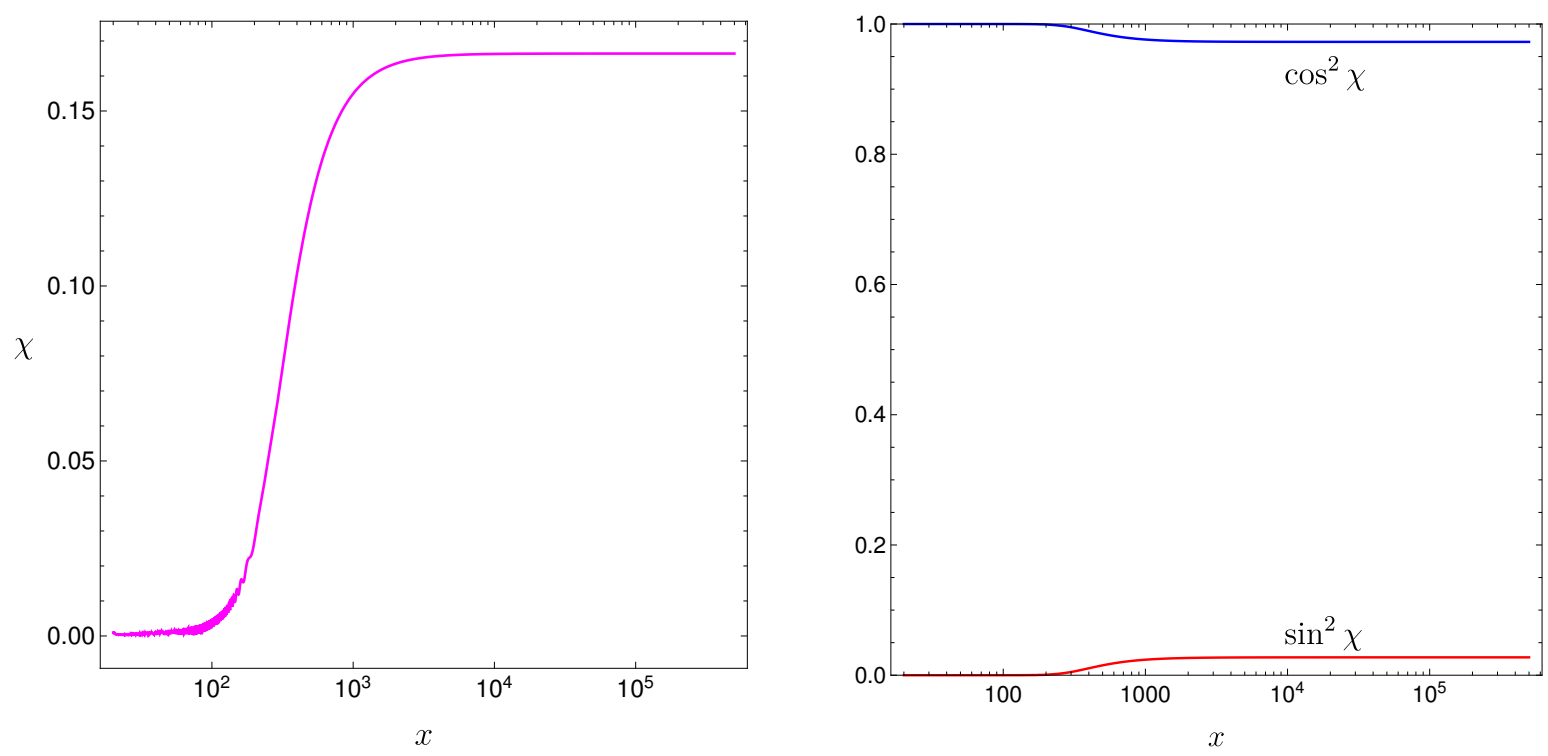

FIG. 1. We show $\chi(x)$ (left panel) and $\cos ^{2}[\chi(x)]$ and $\sin ^{2}[\chi(x)]$ (right panel, blue and red curve respectively) as a function of the dimensionless distance $x$ from the magnetar surface, obtained from a full numerical solution to the evolution equations. The benchmark point is taken to be $\omega=500 \mathrm{keV}, m_{a}=10^{-8} \mathrm{keV}, g_{a \gamma \gamma}=10^{-9} \mathrm{GeV}^{-1}, r_{0}=10 \mathrm{~km}, B_{0}=0.59 \times 10^{14} \mathrm{G}$ and $\theta=\pi / 2$ for Magnetar 1E-2259+586.

actually increases away from the surface. This can be understood from the fact that the photon mass term $\Delta_{\|}$in the denominator in Eq. 11 goes as $\Delta_{\|} \sim 1 / r^{6}$, whereas the ALP-photon mixing term in the numerator goes as $\Delta_{M} \sim 1 / r^{3}$. There is a point around $r \sim \mathcal{O}\left(1000 r_{0}\right)$ where the numerator and denominator become comparable, resulting in a large mixing angle. The probability of conversion becomes large at this position, which we call the radius of conversion $r_{a \rightarrow \gamma}$. Beyond $r_{a \rightarrow \gamma}$, the mixing angle again becomes small since the ALP mass term $\Delta_{a}$ in the denominator of Eq. 11 dominates over both $\Delta_{\|}$as well as $\Delta_{M}$.

We note that a phase resolved analysis will require the introduction of a viewing angle and a time-dependent rotational phase that is related to the magnetar angular velocity. If one assumes that the emission region is localized on the magnetar surface, an opening angle will also be introduced. The spectrum will therefore be functions of these extra parameters, and it is possible that a careful investigation of phase-resolved data will yield constraints stronger than the ones we are achieving in the current work. We leave this analysis for future work.

We briefly comment on the subsequent propagation of unconverted ALPs after they leave the magnetosphere. ALPs with masses $\lesssim 10^{-12} \mathrm{eV}$ emanating from the magnetars in our sample can convert to photons in the magnetic field of the Milky Way and this may yield constraints on $g_{a \gamma \gamma}$. Such constraints depend on several astrophysical parameters, such as the coherent and random magnetic fields, electron density, the distance of the source, the exact value of the Galactic magnetic field, the clumpiness of the interstellar medium, and the warm ionized medium and the warm neutral medium. A full study of these effects may be interesting. We refer to [22, 23] for further details of these topics.

\section{NUCLEON BREMSSTRAHLUNG AND ALP PRODUCTION}

In this section, we outline our calculation of the predicted photon luminosity coming from ALP-photon conversions, which we denote by $L_{a \rightarrow \gamma}$. The observed luminosity of photons produced by the conversion process can be schematically written as

$$
L_{a \rightarrow \gamma}=(\text { production of } a) \times P_{a \rightarrow \gamma},
$$

where $P_{a \rightarrow \gamma}$ is the conversion probability calculated earlier.

The production in the magnetar core proceeds via bremsstrahlung from neutrons $\psi_{n}: \psi_{n}+\psi_{n} \leftrightarrow \psi_{n}+$ $\psi_{n}+a$. The coupling term in the Lagrangian is $\mathcal{L}=$ $G_{a n} \partial_{\mu} a \bar{\psi}_{n} \gamma^{\mu} \gamma_{5} \psi_{n}$ [13. The interaction between the spectator nucleon and the nucleon emitting the axion is modeled by one-pion exchange (OPE) with Lagrangian $\mathcal{L}_{n \pi}=i\left(2 m_{n} / m_{\pi}\right) f \gamma^{5} \pi_{0} \bar{\psi}_{n} \psi_{n}$, where $f \approx 1$. We refer to [24], 25] and references therein for more details. The relevant tree-level Feynman diagrams are given in [13.

The photon luminosity from axion conversion is [26]:

$$
L_{a \rightarrow \gamma}=\int_{0}^{\infty} d \omega \frac{1}{2 \pi} \int_{0}^{2 \pi} d \theta \cdot \omega \cdot \frac{d N_{a}}{d \omega} \cdot P_{a \rightarrow \gamma}(\omega, \theta),
$$

where $N_{a}$ is the axion emission rate (number per time) 
and $d N_{a} / d \omega$ is the axion energy spectrum:

$$
\frac{d N_{a}}{d \omega}=\frac{N_{a}}{T} \frac{x^{2}\left(x^{2}+4 \pi^{2}\right) e^{-x}}{8\left(\pi^{2} \zeta_{3}+3 \zeta_{5}\right)\left(1-e^{-x}\right)},
$$

where $x=\omega / T$ and is a dimensionless quantity. The total emission rate of ALPs $N_{a}$ can be obtained from the following emissivity formula 26]

$$
\begin{aligned}
Q=1.3 \times 10^{19} \mathrm{erg} & \cdot \mathrm{s}^{-1} \cdot \mathrm{cm}^{-3}\left(\frac{G_{a n}}{10^{-10} \mathrm{GeV}^{-1}}\right)^{2} \\
& \times\left(\frac{\rho}{\rho_{0}}\right)^{1 / 3}\left(\frac{T}{10^{9} K}\right)^{6}
\end{aligned}
$$

which is the axion emission rate per volume. Here $\rho$ is the magnetar density and $\rho_{0}=2.8 \times 10^{14} \mathrm{~g} \cdot \mathrm{cm}^{-3}$ is the nuclear saturation density. For a magnetar with radius $r$, the axion emission rate is then given by

$$
\int_{0}^{\infty} d \omega \omega \frac{d N_{a}}{d \omega}=Q \times \frac{4}{3} \pi r^{3}
$$

which is proportional to $G_{a n}^{2}$. For the range of ALPphoton couplings $g_{a \gamma \gamma}$ we are interested in, we can use the semi-analytic expression for the conversion probability given in Eq. 10. Then, it is clear that $P_{a \rightarrow \gamma} \propto g_{a \gamma \gamma}^{2}$. It then follows that $L_{a \rightarrow \gamma} \propto G_{a n}^{2} g_{a \gamma \gamma}^{2}$. Assuming the distance of the magnetar is $d$, then the $\nu F_{\nu}$ spectrum is given by

$$
\nu F_{\nu}(\omega)=\omega^{2} \frac{1}{4 \pi d^{2}} \frac{1}{\omega} \frac{d L_{a \rightarrow \gamma}}{d \omega}
$$

and we choose the unit $\mathrm{MeV}^{2} \mathrm{~cm}^{-2} \mathrm{~s}^{-1} \mathrm{MeV}^{-1}$.

\section{ALP EMISSIVITY IN MEAN FIELD THEORY}

In this section, we discuss the steps involved in the calculation of the ALP emissivity $Q$ from a magnetar core in mean field theory, following the results recently obtained in [27. Although we do not use this more sophisticated treatment for the production process in this paper, we include this discussion for completeness and for use in future work.

To be specific, the discussion will model the nuclear matter inside a neutron star with the $N L \rho$ EoS 28], which is a relativistic mean field theory where nucleons interact by exchanging the scalar $\sigma$ meson and the $\omega$ and $\rho$ vector mesons. Our EoS supports a neutron star of mass $2 M_{\odot}$ with pressure consistent with GW170817 and NICER data for posterior distributions of the pressure at $0.5,1,2,3$ times nuclear saturation density.
In the mean field approximation, we can take the neutron and proton as free particles with effective Dirac masses given by $m_{*}=m-g_{\sigma} \sigma$ and with effective chemical potentials $\mu_{n}^{*}=\mu_{n}-U_{n}$ and $\mu_{p}^{*}=\mu_{p}-U_{p}$. Here, $U_{i}$ are the nuclear mean fields

$$
\begin{aligned}
& U_{n}=g_{\omega} \omega_{0}-\frac{1}{2} g_{\rho} \rho_{03}, \\
& U_{p}=g_{\omega} \omega_{0}+\frac{1}{2} g_{\rho} \rho_{03} .
\end{aligned}
$$

The chemical potentials $\mu_{i}$ and $\mu_{i}^{*}$ are relativistic and contain the rest mass of the particle. The energy dispersion relations are given by

$$
\begin{aligned}
& E_{n}=\sqrt{p^{2}+m_{*}^{2}}+U_{n}, \\
& E_{p}=\sqrt{p^{2}+m_{*}^{2}}+U_{p} .
\end{aligned}
$$

Note that they have been modified by the presence of the nuclear mean field and that the $\rho$ meson distinguishes the neutron from the proton by creating a difference in mean field experienced by the respective particles.

The formalism for calculating the rate of particle processes is given in 29], which uses parameter set I of the model in 28]. For the calculations, the energies in the matrix element should use $E^{*} \equiv \sqrt{p^{2}+m_{*}^{2}}$, while the energy factors in the delta functions and Fermi-Dirac factors should use $E=E^{*}+U_{n}$. The emissivity is given by 13

$$
\begin{aligned}
& Q=\int \frac{d^{3} p_{1}}{(2 \pi)^{3}} \frac{d^{3} p_{2}}{(2 \pi)^{3}} \frac{d^{3} p_{3}}{(2 \pi)^{3}} \frac{d^{3} p_{4}}{(2 \pi)^{3}} \frac{d^{3} \omega}{(2 \pi)^{3}} \frac{S \sum|\mathcal{M}|^{2}}{2^{5} E_{1}^{*} E_{2}^{*} E_{3}^{*} E_{4}^{*} \omega} \omega \\
& \times(2 \pi)^{4} \delta^{4}\left(p_{1}+p_{2}-p_{3}-p_{4}-\omega\right) f_{1} f_{2}\left(1-f_{3}\right)\left(1-f_{4}\right) .
\end{aligned}
$$

Here, the $p_{i}$ and $E_{i}$ are the momenta of the nucleons participating in the Feynman diagram. The Fermi-Dirac factors are given by $f_{i}=\left(1+e^{\left(E_{i}-\mu_{n}\right) / T}\right)^{-1}$. The matrix element is given by

$$
\begin{aligned}
S \sum_{\text {spins }}|\mathcal{M}|^{2} & =\frac{256}{3} \frac{f^{4} m_{n}^{4} G_{a n}^{2}}{m_{\pi}^{4}}\left[\frac{\mathbf{k}^{4}}{\left(\mathbf{k}^{2}+m_{\pi}^{2}\right)^{2}}\right. \\
& \left.+\frac{\mathbf{l}^{4}}{\left(\mathbf{l}^{2}+m_{\pi}^{2}\right)^{2}}+\frac{\mathbf{k}^{2} \mathbf{l}^{2}-3(\mathbf{k} \cdot \mathbf{l})^{2}}{\left(\mathbf{k}^{2}+m_{\pi}^{2}\right)\left(\mathbf{l}^{2}+m_{\pi}^{2}\right)}\right]
\end{aligned}
$$

where $\mathbf{k}$ and $\mathbf{l}$ are three-momentum transfers $\mathbf{k}=\mathbf{p}_{\mathbf{2}}-\mathbf{p}_{\mathbf{4}}$ and $\mathbf{l}=\mathbf{p}_{\mathbf{2}}-\mathbf{p}_{\mathbf{3}}$. The symmetry factor for these diagrams is $S=1 / 4$.

We outline four different regimes in which we compute $Q$. The first is relativistic matter with arbitrary degeneracy in the Fermi surface approximation, when neutrons are strongly degenerate, in which case only neutrons near the Fermi surface participate in the bremsstrahlung process. The axion emissivity is

$$
Q_{F S}=\frac{31}{2835 \pi} \frac{f^{4} G_{a n}^{2} m_{n}^{4}}{m_{\pi}^{4}} p_{F n} F(y) T^{6},
$$


where

$$
\begin{aligned}
F(y)= & 4-\frac{1}{1+y^{2}}+\frac{2 y^{2}}{\sqrt{1+2 y^{2}}} \arctan \left(\frac{1}{\sqrt{1+2 y^{2}}}\right) \\
& -5 y \arcsin \left(\frac{1}{\sqrt{1+y^{2}}}\right),
\end{aligned}
$$

with $y=m_{\pi} /\left(2 p_{F n}\right)$.

The Fermi surface approximation extends the lower endpoint of integration of neutron energy to $-\infty$. An improvement to the Fermi surface approximation can be obtained, which keeps the neutron energy bounded by $m_{*}+U_{n}<E_{n}<\infty$. The ALP emissivity in this im- proved approximation is 27 .

$$
Q_{F S, \text { improved }}=\frac{2}{3 \pi^{7}} \frac{f^{4} G_{a n}^{2} m_{n}^{4}}{m_{\pi}^{4}} p_{F n} F(y) T^{6} K_{2}(\hat{y}),
$$

where

$$
\begin{aligned}
K_{2}(\hat{y}) & =\int_{-2 \hat{y}}^{\infty} d u \frac{1}{1-e^{u}} \ln \left\{\frac{\cosh (\hat{y} / 2)}{\cosh [(u+\hat{y}) / 2]}\right\} \\
& \times \int_{0}^{u+2 \hat{y}} d w \frac{w^{2}}{1-e^{w-u}} \ln \left\{\frac{\cosh [(u+\hat{y}-w) / 2]}{\cosh (\hat{y} / 2)}\right\} .
\end{aligned}
$$

The third approximation we discuss assumes nonrelativistic neutrons. The full momentum dependence of the matrix element in Eq. 23 is retained when evaluating the emissivity from Eq. 22 The expression obtained in this case is 27 .

$$
\begin{aligned}
Q_{n o n-r e l} & =\frac{32 \sqrt{2}}{3 \pi^{8}} \frac{f^{4} m_{n}^{4} G_{a n}^{2}}{m_{\pi}^{4}} m_{*}^{1 / 2} T^{6.5} \int_{0}^{\infty} d u d v \int_{0}^{v} d w \int_{-1}^{1} d r d s \int_{0}^{2 \pi} d \phi u^{1 / 2} v^{3 / 2} w^{3 / 2}(v-w)^{2} \\
& \times \frac{\left(\alpha^{4}\left(r^{2}+3\right)-6 \alpha^{2}\left(r^{2}-1\right)(v+w)-3\left(r^{2}-1\right)\left(2\left(1-2 r^{2}\right) v w+v^{2}+w^{2}\right)\right)}{\left[2 w\left(\alpha^{2}-2 r^{2} v+v\right)+\left(\alpha^{2}+v\right)^{2}+w^{2}\right]^{2}} \\
& \times\left[\left(1+e^{\beta\left(E_{1}-\mu_{n}\right)}\right)\left(1+e^{\beta\left(E_{2}-\mu_{n}\right)}\right)\left(1+e^{-\beta\left(E_{3}-\mu_{n}\right)}\right)\left(1+e^{-\beta\left(E_{4}-\mu_{n}\right)}\right)\right]^{-1},
\end{aligned}
$$

where $\alpha=m_{\pi} / \sqrt{2 m_{*} T}$. This integral can be performed numerically.

The final approximation we discuss involves a calcula- tion of the fully relativistic phase-space integration in Eq. 22 performed with a constant matrix element in Eq.223 The result is (we refer to 27] for a full derivation)

$$
\begin{aligned}
Q_{r e l} & =\left(1-\frac{\beta}{3}\right) \frac{f^{4} m_{n}^{4} G_{a n}^{2}}{8 \pi^{7} m_{\pi}^{4}}\left(1+\frac{m_{\pi}^{2}}{k_{\text {typ }}^{2}}\right)^{-2} \int_{m_{*}}^{\infty} d q_{0} \int_{0}^{\infty} d q \int_{0}^{\sqrt{q_{0}^{2}-m_{*}^{2}}} d k \int_{m_{*}}^{q_{0}} d l_{0} \int_{0}^{\sqrt{l_{0}^{2}-m_{*}^{2}}} d l \int_{\omega_{-}\left(l_{0}, l\right)}^{\omega_{+}\left(l_{0}, l\right)} d \omega \\
& \times k \omega\left(q_{0}-\sqrt{k^{2}+m_{*}^{2}}\right) \frac{\theta\left(2 k q-\left|q_{0}^{2}-q^{2}-2 q_{0} \sqrt{k^{2}+m_{*}^{2}}\right|\right) \theta\left(2 q l-\left|m_{*}^{2}+q^{2}+l^{2}-q_{0}^{2}-l_{0}^{2}+2 q_{0} l_{0}\right|\right)}{\sqrt{k^{2}+m_{*}^{2}} \sqrt{k^{2}+m_{*}^{2}+q_{0}^{2}-2 q_{0} \sqrt{k^{2}+m_{*}^{2}}}} \\
& \times\left[\left(1+e^{\left(\sqrt{k^{2}+m_{*}^{2}}-\mu_{n}^{*}\right) / T}\right)\left(1+e^{\left(\sqrt{k^{2}+m_{*}^{2}+q_{0}^{2}-2 q_{0} \sqrt{k^{2}+m_{*}^{2}}}-\mu_{n}^{*}\right) / T}\right)\left(1+e^{-\left(q_{0}-l_{0}-\mu_{n}^{*}\right) / T}\right)\left(1+e^{-\left(l_{0}-\omega-\mu_{n}^{*}\right) / T}\right)\right]^{-1} .
\end{aligned}
$$

This integral can also be performed numerically.

The emissivities resulting from the four approximations described were compared in [27, and it was found that they show remarkable convergence for temperatures $T \lesssim 10 \mathrm{MeV}$, which is the regime we are mainly interested in for the magnetar core. Using these results, one can calculate the normalized ALP spectrum and ALP emissivity to yield a constraint on the product
$G_{a n} \times g a \gamma \gamma$. We leave this for future work.

\section{CALCULATION OF REFRACTIVE INDICES}

In this section, we provide general expressions for the photon refractive indices in the parallel and perpendicular directions. We are interested in several different 
regimes of the photon frequency and the strength of the external magnetic field:

(i) $\omega \ll 2 m_{e}$ and $B \ll B_{c}$ : soft X-rays in an external magnetic field that is much weaker than the critical strength. This regime is relevant for the conversion of less energetic ALPs into photons at the radius of conversion $\sim 500 r_{0}$, where $B \sim 10^{-5} B_{c}$. Since the photon energies are much smaller than $m_{e}$, the Euler-Heisenberg approximation can be used to calculate the refractive indices.

(ii) $\omega \lesssim 2 m_{e}$ and $B \ll B_{c}$, with $\left(\frac{\omega}{2 m_{e}}\right)^{2}\left(\frac{B}{B_{c}}\right)^{2} \ll 1$ : hard X-rays and soft gamma-rays in an external magnetic field that is much weaker than the critical strength. This regime is relevant for the conversion of energetic ALPs with $\omega \sim \mathcal{O}(100) \mathrm{keV}-\mathcal{O}(1) \mathrm{MeV}$ into photons at the radius of conversion $\sim 500 r_{0}$, where $B \sim 10^{-5} B_{c}$. This regime is relevant for the observational signatures considered in this paper.

(iii) $\omega \lesssim 2 m_{e}$ and $B>B_{c}$ : hard X-rays and soft gamma-rays in an external magnetic field that is stronger than the critical strength. This regime is relevant for the conversion of energetic ALPs with $\omega \sim \mathcal{O}(100) \mathrm{keV}-$ $\mathcal{O}(1) \mathrm{MeV}$ into photons from the magnetar surface to a distance of $\sim 3 r_{0}$.

We now turn to a discussion of the refractive indices in regimes $(i i)$ and $(i i i)$, which are relevant for this paper.

Quantum corrections to the photon propagator can be studied using the photon polarization tensor $\Pi^{\mu \nu}$, defined in the following way [30]

$$
\mathcal{L} \supset-\frac{1}{2} \int_{x^{\prime}} A_{\mu}(x) \Pi^{\mu \nu}\left(x, x^{\prime}\right) A_{\nu}\left(x^{\prime}\right),
$$

where $A_{\mu}$ is the propagating photon. To evaluate $\Pi^{\mu \nu}$, we can consider the perpendicular and parallel components of the momentum four-vector $k^{\mu}$. We note that these components are defined with respect to the external magnetic field $\vec{B}$, which we take to point in the direction $\vec{e}_{1}: k^{\mu}=k_{\|}^{\mu}+k_{\perp}^{\mu}, k_{\|}^{\mu}=\left(\omega, k^{1}, 0,0\right)$, and $k_{\perp}^{\mu}=\left(0,0, k^{2}, k^{3}\right)$. The metric tensor can likewise be decomposed into the parallel and perpendicular directions: $g^{\mu \nu}=g_{\|}^{\mu \nu}+g_{\perp}^{\mu \nu}$, where $g_{\|}^{\mu \nu}=\operatorname{diag}(-1,+1,0,0)$ and $g_{\perp}^{\mu \nu}=\operatorname{diag}(0,0,+1,+1)$.

We will assume a pure and homogeneous external magnetic field to work out the photon polarization tensor, since taking into account the spatial variation of the magnetic field would be significantly more complicated. This is justified, since the dipolar magnetic field varies at a scale given by the magnetar radius, while the photon wavelength is much smaller in the soft gamma-ray regime. At one loop, the polarization tensor is given by 31, 34,

$$
\begin{aligned}
\Pi^{\mu \nu}(k)= & \frac{\alpha}{2 \pi} \int_{-1}^{1} \frac{\mathrm{d} \nu}{2} \int_{0}^{\infty-\mathrm{i} \eta} \frac{\mathrm{d} s}{s}\left\{\mathrm { e } ^ { - \mathrm { i } \Phi _ { 0 } s } \left[-N_{0} k^{\mu} k^{\nu}\right.\right. \\
& +\left(N_{1}-N_{0}\right)\left(g_{\|}^{\mu \nu} k_{\|}^{2}-k_{\|}^{\mu} k_{\|}^{\nu}\right) \\
& \left.+\left(N_{2}-N_{0}\right)\left(g_{\perp}^{\mu \nu} k_{\perp}^{2}-k_{\perp}^{\mu} k_{\perp}^{\nu}\right)\right] \\
& \left.+\left(1-\nu^{2}\right) \mathrm{e}^{-\mathrm{i}\left(m_{e}^{2}-\mathrm{i} \epsilon\right) s} k^{\mu} k^{\nu}\right\}
\end{aligned}
$$

where $\Phi_{0}=m_{e}^{2}-\mathrm{i} \epsilon+n_{1} k_{\|}^{2}+n_{2} k_{\perp}^{2}, s$ is the proper time, $\nu$ governs the loop momentum distribution, and $\epsilon$ and $\eta$ are parameters that tend to $0^{+}$. The external magnetic field appears in the scalar functions $N_{0}, N_{1}, N_{2}, n_{1}$ and $n_{2}$. In terms of the variable $z=e B s$, these functions are given by

$$
\begin{aligned}
& N_{0}(z)=\frac{z}{\sin z}(\cos \nu z-\nu \sin \nu z \cot z), \quad n_{1}(z)=\frac{1-\nu^{2}}{4} \\
& N_{1}(z)=z\left(1-\nu^{2}\right) \cot z, \quad n_{2}(z)=\frac{\cos \nu z-\cos z}{2 z \sin z} \\
& N_{2}(z)=\frac{2 z(\cos \nu z-\cos z)}{\sin ^{3} z}
\end{aligned}
$$

The polarization tensor is most compactly expressed in terms of the projection operators $P_{\|}^{\mu \nu}$ and $P_{\perp}^{\mu \nu}$, defined in the following way

$$
P_{\|}^{\mu \nu}=g_{\|}^{\mu \nu}-\frac{k_{\|}^{\mu} k_{\|}^{\nu}}{k_{\|}^{2}} \quad \text { and } \quad P_{\perp}^{\mu \nu}=g_{\perp}^{\mu \nu}-\frac{k_{\perp}^{\mu} k_{\perp}^{\nu}}{k_{\perp}^{2}},
$$

in terms of which the tensor can be re-expressed as 31

$$
\Pi^{\mu \nu}(k)=P_{\|}^{\mu \nu} \Pi_{\|}+P_{\perp}^{\mu \nu} \Pi_{\perp},
$$

where

$$
\left\{\begin{array}{l}
\Pi_{\|} \\
\Pi_{\perp}
\end{array}\right\}=\frac{\alpha}{2 \pi} \int_{-1}^{1} \frac{\mathrm{d} \nu}{2} \int_{0}^{\infty-\mathrm{i} \eta} \frac{\mathrm{d} s}{s}\left[\mathrm{e}^{-\mathrm{i} \Phi_{0} s}\left\{\begin{array}{l}
k_{\|}^{2} N_{1}+k_{\perp}^{2} N_{0} \\
k_{\|}^{2} N_{0}+k_{\perp}^{2} N_{2}
\end{array}\right\}\right] .
$$

The expression in Eq. 35 is amenable to a perturbative expansion, which we now explore.

$$
\text { A. } \omega \lesssim 2 m_{e} \text { and } B \ll B_{c} \text {, with }\left(\frac{\omega}{2 m_{e}}\right)^{2}\left(\frac{B}{B_{c}}\right)^{2} \ll 1
$$

We first note that a perturbative expansion of $\Pi_{p}^{\text {pert }}$ (where $p=\|, \perp$ ) in powers of the magnetic field can be obtained by an expansion in powers of $(e B)^{2 n}$ :

$$
\Pi_{p}^{\text {pert }}=\sum_{n=0}^{\infty} \Pi_{p}^{(2 n)}
$$


with the even powers being due to Furry's theorem, and

$$
\Pi_{p}^{(2 n)}=\frac{(e B)^{2 n}}{n !}\left[\left(\frac{\partial}{\partial(e B)^{2}}\right)^{n} \Pi_{p}\right]_{e B=0},
$$

Since the limit $z \rightarrow 0$ does not admit any poles in the complex $s$ plane for the integrands, the integration over $s$ can be performed on the real positive axis. This yields the following expressions for the $\Pi_{p}^{(2 n)}[35$ :

$$
\begin{aligned}
& \left\{\begin{array}{l}
\Pi_{\|}^{(2 n)} \\
\Pi_{\perp}^{(2 n)}
\end{array}\right\}=\frac{\alpha}{2 \pi} \int_{-1}^{1} \frac{\mathrm{d} \nu}{2} \int_{0}^{\infty} \frac{\mathrm{d} s}{s} \mathrm{e}^{-\mathrm{i} \phi_{0} s} \frac{z^{2 n}}{n !} \\
& \times\left[\left(\frac{\partial}{\partial z^{2}}\right)^{n}\left(\left\{\begin{array}{c}
k_{\|}^{2} N_{1}+k_{\perp}^{2} N_{0} \\
k_{\|}^{2} N_{0}+k_{\perp}^{2} N_{2}
\end{array}\right\} \mathrm{e}^{-\mathrm{i} s k_{\perp}^{2} \tilde{n}_{2}}\right)\right]_{z=0},
\end{aligned}
$$

where $\tilde{n}_{2}=n_{2}-\frac{1-\nu^{2}}{4}=\mathcal{O}\left(z^{2}\right)$.

The integral over $s$ in Eq. 38 can be performed explicitly. Using the expressions in Eq. 32, one obtains

$$
\begin{aligned}
\Pi_{p}^{(2 n)}= & \frac{\alpha}{2 \pi} \int_{-1}^{1} \frac{\mathrm{d} \nu}{2} \sum_{l=0}^{n-1} \frac{(2 n+l-1) !}{(-1)^{n+l}}\left[k_{\|}^{2} c_{p}^{\|(n, l)}\left(\nu^{2}\right)\right. \\
& \left.+k_{\perp}^{2} c_{p}^{\perp(n, l)}\left(\nu^{2}\right)\right]\left(\frac{e B}{m_{e}^{2}}\right)^{2 n}\left(\frac{k_{\perp}^{2}}{m_{e}^{2}}\right)^{l},
\end{aligned}
$$

where the coefficients $c_{p}^{\|(n, l)}\left(\nu^{2}\right)$ and $c_{p}^{\perp(n, l)}\left(\nu^{2}\right)$ can be obtained explicitly from expanding Eq. 32 .

We note that a perturbative expansion can be obtained when both expansion parameters in Eq. 39 are small:

$$
\frac{e B}{m_{e}^{2}} \equiv \frac{B}{B_{c}} \ll 1 \quad \text { and } \quad\left(\frac{B}{B_{c}}\right)^{2} \frac{\omega^{2} \sin ^{2} \theta}{m_{e}^{2}} \ll 1,
$$

where we have introduced the angle $\theta$ between the magnetic field and the photon propagation direction. The leading order tensor is

$$
\begin{aligned}
\left\{\begin{array}{c}
\Pi_{\|}^{(2)} \\
\Pi_{\perp}^{(2)}
\end{array}\right\}= & -\frac{\alpha}{12 \pi} \int_{-1}^{1} \frac{\mathrm{d} \nu}{2}\left(\frac{e B}{\phi_{0}}\right)^{2}\left(1-\nu^{2}\right)^{2} \\
& \times\left[\left\{\begin{array}{c}
\frac{-2}{1-\nu^{2}} \\
1
\end{array}\right\} k_{\|}^{2}+\left\{\begin{array}{c}
1 \\
\frac{5-\nu^{2}}{2\left(1-\nu^{2}\right)}
\end{array}\right\} k_{\perp}^{2}\right] .
\end{aligned}
$$

The integration over $\nu$ finally yields 36 and 37 ]

$$
\left\{\begin{array}{l}
\Pi_{\|}^{(2)} \\
\Pi_{\perp}^{(2)}
\end{array}\right\}=-\frac{\alpha}{2 \pi}\left(\frac{B}{B_{c}}\right)^{2} \omega^{2} \sin ^{2} \theta \frac{2}{45}\left\{\begin{array}{l}
7 \\
4
\end{array}\right\},
$$

The corresponding indices of refraction are given by $n_{p}=1-\frac{1}{2 \omega^{2}} \Re\left(\Pi_{p}\right)$, which yields

$$
\left\{\begin{array}{l}
n_{\|} \\
n_{\perp}
\end{array}\right\}=1+\frac{\alpha}{4 \pi}\left(\frac{B}{B_{c}}\right)^{2} \sin ^{2} \theta \frac{2}{45}\left\{\begin{array}{l}
7 \\
4
\end{array}\right\}+\mathcal{O}\left((e B)^{4}\right) .
$$

$$
\text { B. } \omega \lesssim 2 m_{e} \text { and } B>B_{c}
$$

This regime is relevant for the conversion of energetic ALPs with $\omega \sim \mathcal{O}(100) \mathrm{keV}-\mathcal{O}(1) \mathrm{MeV}$ into photons from the magnetar surface to a distance of $\sim 3 r_{0}$. We only quote the final answer here, referring to [38] for a full derivation:

$$
\begin{aligned}
& \left\{\begin{array}{l}
n_{\|} \\
n_{\perp}
\end{array}\right\}=1+\frac{\alpha}{4 \pi} \sin ^{2} \theta\left[\left(\frac{2}{3} \frac{B}{B_{c}}-\Sigma\right)\left\{\begin{array}{l}
1 \\
0
\end{array}\right\}\right. \\
& \left.-\left[\frac{2}{3}+\frac{B_{c}}{B} \ln \left(\frac{B_{c}}{B}\right)\right]\left\{\begin{array}{c}
1 \\
-1
\end{array}\right\}+\mathcal{O}\left(\frac{1}{e B}\right)+\mathcal{O}\left(\omega^{2}\right)\right] .
\end{aligned}
$$

Here, $\Sigma \sim \mathcal{O}(1)$ is a constant.

\section{SOFT GAMMA-RAY BACKGROUND}

Magnetars exhibit thermal X-ray emission below 10 $\mathrm{keV}$ and a hard pulsed non-thermal X-ray emission with power law tails above $10 \mathrm{keV}$. This hard X-ray emission can extend to between $150-275 \mathrm{keV}$ [39 41] and appears to turn over above $275 \mathrm{keV}$ due to ULs being obtained with INTEGRAL SPI (20-1000 keV) and CGRO COMPTEL $(0.75-30 \mathrm{MeV})$ 18. A spectral break above $1 \mathrm{MeV}$ is also inferred by the non-detection of 20 magnetars using Fermi-LAT above $100 \mathrm{MeV}$ [2]. The hard Xray emission is most likely caused by resonant Compton upscattering (RCU) of surface thermal X-rays by nonthermal electrons moving along the magnetic field lines of the magnetosphere. The initial modeling of 43, using $B$ field strengths typical of magnetars, at three times the quantum critical field strength $B_{c}$ produces flat differential flux spectra with sharp cut-offs at energies directly proportional to the electron Lorentz factor $\left(\gamma_{\mathrm{e}}\right)$ and places the maximum extent of the Compton resonasphere within a few stellar radii of the magnetar surface.

In [44, Monte-Carlo models of the RCU of soft thermal photons, incorporating the relativistic QED resonant cross section, produces flat spectra up to $1 \mathrm{MeV}$ for highly relativistic electrons $\left(\gamma_{\mathrm{e}}=22\right)$, whilst mildly relativistic electrons $\left(\gamma_{\mathrm{e}}=1.7\right)$ demonstrate spectral breaks at $316 \mathrm{keV}$. In [45], an analytic model of RCU, considering relativistic particle injection $\left(\gamma_{\mathrm{e}}>>10\right)$ and deceleration within magnetic loops predicts a spectral peak at $\sim 1$ $\mathrm{MeV}$ and a narrow annihilation line at $511 \mathrm{keV}$ (both as yet unobserved). This model also places the active field loops emitting photons at 3-10 stellar radii for a surface $B$ field of $\sim 10^{15} \mathrm{G}$.

The analysis of [43] is recently extended in 46, allowing for a QED Compton cross scattering section which incorporates spin-dependent effects in stronger $B$ fields. Electrons with energies $\lesssim 15 \mathrm{MeV}$ will emit most energy below $250 \mathrm{keV}$ which is consistent with the hard inferred $\mathrm{X}$-ray turnover above. In [46, the maximum resonant cut-off energy can reach a peak of $810 \mathrm{keV}$, for $\gamma_{\mathrm{e}}=10$ 
at some magnetar rotational phases and viewing angles which violates COMPTEL ULs, however the model neglects the effects of Compton cooling and attenuation processes such as photon absorption due to magnetic pair creation $\left(\gamma \rightarrow e^{+} e^{-}\right)$and photon splitting $(\perp \rightarrow\|\|)$. Also, the effect of electron Compton cooling is expected to steepen the cut-offs seen in the predicted hard X-ray spectral tails and allow the models to then be in agreement with the COMPTEL ULs. The emission region is placed at $4-15$ and $2.5-30$ stellar radii for $\gamma_{\mathrm{e}}$ values of 10 and 100 respectively.

The attenuation processes of magnetic pair creation and photon splitting which act to suppress photon emission in RCU are considered in detail in [47. for typical magnetar surface $B$ fields of $10 B_{c}$. In this case, the photon splitting opacity alone constrains the emission region of observed $250 \mathrm{keV}$ emission in magnetars to be outside altitudes of 2-4 stellar radii and photons emitted from the magnetar surface at magnetic co-latitudes $<20^{\circ}$ can escape with energies $>1 \mathrm{MeV}$ for typical magnetar surface $B$ fields of $10 B_{c}$. Also the emission of photons from field loops at $<2$ stellar radii is suppressed with photon escape energies of no greater then $287 \mathrm{keV}$. In contrast, emission regions at altitudes of $>5$ stellar radii guarantee escape of $1 \mathrm{MeV}$ photons at nearly all co-latitudes. The photon opacity caused by pair creation is shown to be much less restrictive and does not impact the $<1 \mathrm{MeV}$ band. Finally Fig 8 of reference [47] shows the maximum energies produced by the resonant Compton process alongside the photon escape energies allowed by the photon splitting process (i.e. the maximum photon energies which can escape to an observer) as a function of magnetar rotational phase and obliqueness of rotation (which is the misalignment of the magnetic and rotational axis) and observer angle. It shows that photon emission $>1 \mathrm{MeV}$ is permitted at some but not all rotational phases in the meridional case and that in most cases the RCU emission will vary with rotational phase in the $300 \mathrm{keV}-1 \mathrm{MeV}$ band.

Therefore, the RCU process may produce a background to the signal we wish to measure and this background might be expected to produce pulsed emission when photon opacity due to photon splitting is taken into account. On the other hand, a spectral turn over is possible if the electrons in the magnetosphere field loops are mildly relativistic. In addition, pulsed emission in magnetars has not been observed in the $300 \mathrm{keV}-1$ $\mathrm{MeV}$ band which would be suggestive of an RCU emission mechanism. We also note that photon splitting / pair creation opacity will not attenuate photon emission $<1 \mathrm{MeV}$ at $>10$ stellar radii [47. As axion to photon conversion will occur at $\sim 300$ stellar radii, photon opacity processes can be disregarded. In addition, the 440 magnetar bursts observed with the Fermi-GBM over 5 years have been spectrally soft with typically no emission above $200 \mathrm{keV}$ 48].

A reasonable assumption resulting from the above discussion would be that there is no RCU background and that all emission in the $300 \mathrm{keV}-1 \mathrm{MeV}$ band results from ALP to photon conversion. We instead opt for a slightly more conservative approach and require that any emission from ALP-photon conversion be bounded by the observed emission. This results in ULs on the ALP-photon coupling.

\section{MAGNETAR CORE TEMPERATURES}

We now summarise the need for a magnetar heating mechanism over and above that found in conventional pulsars and discuss temperature modelling which supports the range of values we have chosen for the magnetar core temperature $\left(T_{c}\right)$.

The quiescent X-ray luminosity of magnetars of $10^{34}-10^{35} \mathrm{erg} \mathrm{s}^{-1}$ exceeds the spin down luminosity of $10^{32}-10^{34}$ erg $\mathrm{s}^{-1}$, thus excluding rotation spin down as the sole magnetar energy source. Furthermore, the lack of Doppler modulation in X-ray pulses arising from magnetars indicates a lack of binary companions, which combined with the slow periods of magnetars $(2-12 \mathrm{~s})$ excludes an accretion powered interpretation [49] and 50].

In reference [51, the authors show the need for heating by theoretical cooling curves for neutron stars of mass 1.4 $\mathrm{M}_{\odot}$, with and without proton superfluidity in the core, which yield effective surface temperatures below those observed in seven magnetars (including four in our selection, namely: 1E 1841-045, 1RXS J170849.0-400910, $4 \mathrm{U} 0142+61$ and $1 \mathrm{E} 2259+586$ ). They then use a general relativistic cooling code which accounts for thermal losses from neutrino and photon emission and allows for thermal conduction to show that magnetars are hot inside with $T_{c}=10^{8.4} \mathrm{~K}$ at age $1000 \mathrm{yr}$ and temperatures of $10^{9.1} \mathrm{~K}$ in the crust, where the heat source should be located for efficient warming of the surface, to offset neutrino heat losses from the core.

The authors of 52 consider the case of magnetars born with initial periods of $\leq 3 \mathrm{~ms}$ combined with a strong internal toroidal $B$ field of $\geq 3 \times 10^{16} \mathrm{G}$ and an exterior dipole $B$ field of $\leq 2 \times 10^{1 \overline{4}} \mathrm{G}$. In this case, efficient heating of the core can occur via ambipolar diffusion which has a time varying decay scale as a function of $T_{c}$ and $B$ field strength. As the core cools, an equilibrium is established between increasing $B$ field decay and reducing neutrino emission, leading to reduced cooling which can keep $T_{c}$ at $10^{8.9} \mathrm{~K} 2250 \mathrm{yr}$ after magnetar creation.

The magnetar temperature modeling of [53] considers heating throughout the magnetar core arising from magnetic field decay and ambipolar diffusion, together with the cooling caused by the neutrino emission of the modified URCA process and Cooper pairing of nucleons. In this case, the authors find that strong core heating cannot account for the observed surface temperatures and conclude that, as in the case of [51, high surface temperatures require heating of the crust, rather than the core, with the crust and the core being thermally decoupled from one another. However the authors of [53] show 

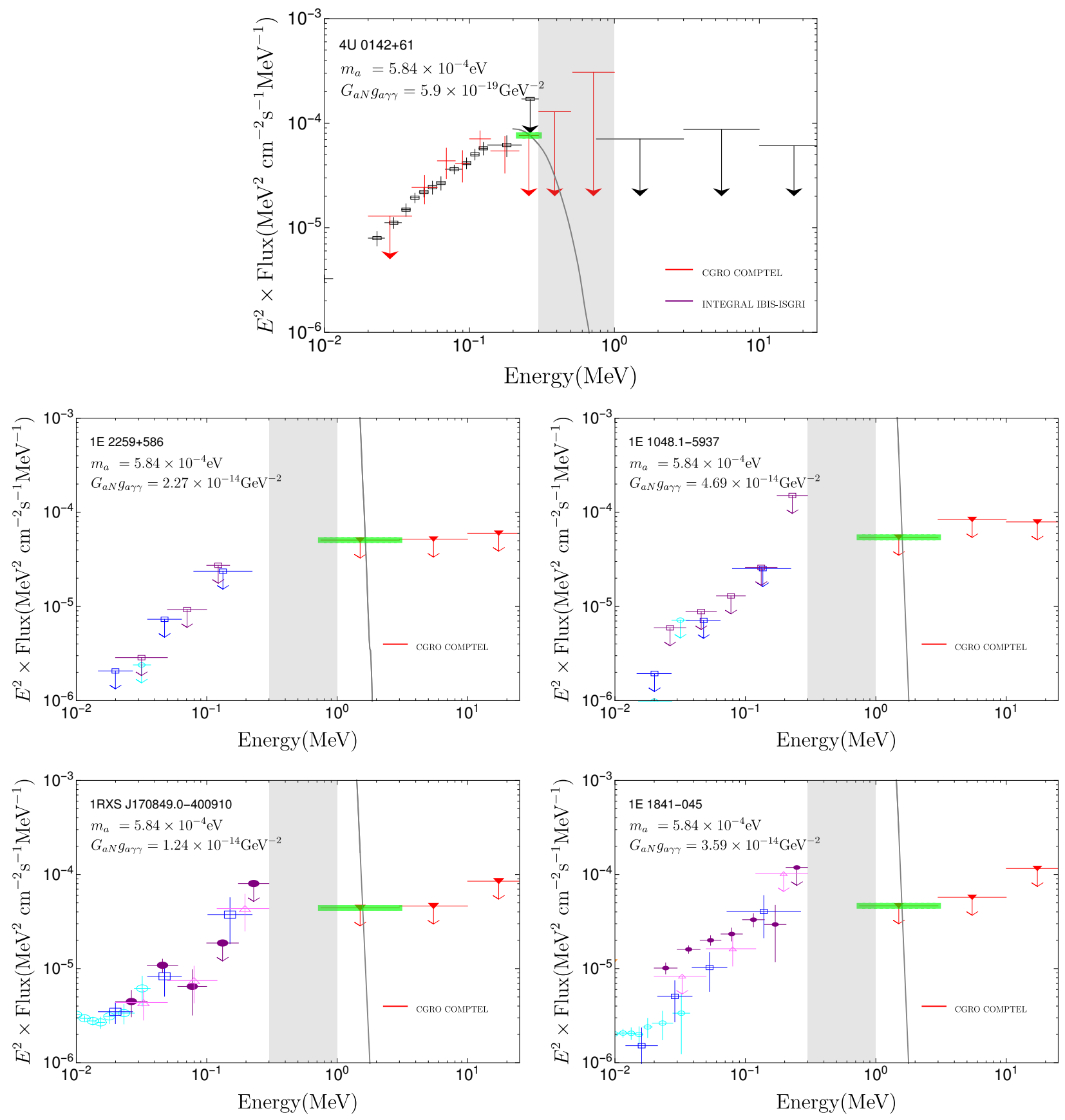

FIG. 2. The spectral energy distributions of the five magnetars from Refs. 16, 18. The ULs within the analysis band, 300 $\mathrm{keV}-1 \mathrm{MeV}$ (grey shaded region, with legend showing relevant experiments) or immediately adjacent to it, are used in the analysis. For each magnetar, the gray line is an example spectrum from axion conversion for the mass and coupling labelled out, which falls on the limit curve in Fig. 3 of the main paper, with the corresponding UL responsible for it denoted by the green thick line.

that $T_{c}$ at $10^{4} \mathrm{yr}$ can vary between $0.8 \times 10^{8} \mathrm{~K}$ with $\boldsymbol{n o}$ heating of the superfluid core, $1.4 \times 10^{8} \mathrm{~K}$ with heating of the crust and $5 \times 10^{8} \mathrm{~K}$ with core heating. At $10^{3} \mathrm{yr}$, with heating of the superfluid core, $T_{c}$ can reach $7 \times 10^{8}$ K.

The strong $B$ field of magnetars can produce strongly anisotropic thermal conductivity in the neutron star crust whilst also allowing the synchrotron neutrino process to become a predominant cooling mechanism while other contributions to the neutrino emissivity are far more weakly suppressed. These effects allow the temperature at the base of the crust heat blanketing envelope to reach $10^{9.6} \mathrm{~K}$ while the surface temperature remains at $10^{5}$ and $10^{6.7} \mathrm{~K}[54$, for a $B$ field parallel and radial to 
the neutron star surface respectively. This is compatible with the observed surface temperatures of $10^{6.5}-10^{6.95}$ $\mathrm{K}$ for the seven magnetars in [51] and could allow $T_{c}$ to exceed $10^{9} \mathrm{~K}$.

Finally, the quiescent luminosity of magnetars $10^{34}-10^{35} \mathrm{erg} \mathrm{s}^{-1}$ implies a $T_{c}$ of $(2.7-\geq 8.0) \times 10^{8} \mathrm{~K}$ for a magnetar with an accreted iron envelope and (1.0 $-5.5) \times 10^{8} \mathrm{~K}$ for an accreted light element envelope 55 .

There are no published $T_{c}$ values for the magnetars in our selection. We therefore study the dependence of our results on a range of core temperatures.

\section{MAGNETAR SELECTION AND UL SOFT-GAMMA-RAY FLUX DETECTION}

We select 5 magnetars which have published ULs for differential energy fluxes between $300 \mathrm{keV}-1 \mathrm{MeV}$ (Table I). These are obtained from the INTEGRAL Soft-Gamma-Ray imager (ISGRI) detector, its Image on Board instrument (IBIS) and spectrometer (SPI); and from the non-contemporaneous observations of the COMPTEL instrument on the Compton Gamma-Ray Observatory CGRO [16] and 18].

We extract UL fluxes from the spectral energy distributions of [16 and 18] using an energy resolved analysis as described in Sec X

\section{SPECTRAL ANALYSIS}

For the five magnetars, the experimental ULs are taken from Ref. [16, 18 and are shown in Fig. 22 We select the ULs which fall within or overlap with the range range $300 \mathrm{keV}-1 \mathrm{MeV}$. For each magnetar and for each axion mass, we require that the spectrum does not exceed any of the ULs on the $\log -\log \nu F_{\nu}$ plot in Fig. 2. The maximal coupling $G_{a n} g_{a \gamma \gamma}$ satisfying above criterion is chosen as the exclusion UL for the coupling product. In comparing the spectrum with each UL in energy bin, say, " $i$ ", we compare the averaged spectrum within that bin with the experimental UL there. More precisely speaking, for each magnetar and each ALP mass, we find the largest coupling $G_{a n} g_{a \gamma \gamma}$ compatible with the following condition:

$$
\frac{\int_{\omega_{i}^{-}<\omega<\omega_{i}^{+}} \log \left[\nu F_{\nu}(\omega)\right] d \log \omega}{\log \omega_{i}^{+}-\log \omega_{i}^{-}} \leq \mathrm{UL}_{i}, \quad \text { for all } i
$$

where $\mathrm{UL}_{i}$ is the upper limit for the $i$-th bin $\left(\omega_{i}^{-}, \omega_{i}^{+}\right)$. This denotes a direct comparison of the photon spectrum from axion conversion with the upper limits in Fig 2, For the most constraining UL, the spectrum at the found coupling product gives the same area as the corresponding UL on the log-log plot. This is illustrated by the example spectrum in each plot (gray curve) for a chosen mass $m_{a}$ and coupling product. Note that the peaks of the gray

\begin{tabular}{cc}
\hline Magnetar & $G_{a n} g_{a \gamma \gamma}\left(\mathrm{GeV}^{-2}\right)$ \\
\hline 1E $2259+586$ & $1.71 \times 10^{-14}$ \\
4U 0142+61 & $2.42 \times 10^{-19}$ \\
1RXS J170849.0-400910 & $2.20 \times 10^{-15}$ \\
1E 1841-045 & $1.64 \times 10^{-14}$ \\
1E 1048.1-5937 & $8.44 \times 10^{-15}$ \\
\hline
\end{tabular}

TABLE II. Results: The $95 \%$ CL UL on the product of couplings $G_{a n} \times g_{a \gamma \gamma}$ obtained from conversions for the magnetars in our sample. The ALP mass is chosen to be $10^{-7} \mathrm{eV}$ for all benchmarks shown in this table. The assumed $T_{c}$ is $5 \times 10^{8}$ K.

line are at around $0.2 \mathrm{KeV}$ for all example spectra shown, and for the four magnetars excluding $4 \mathrm{U} 0142+61$, the peaks are at a much higher amplitude, outside the plot range of these plots. For the example spectra in each plot, the corresponding most constraining UL is highlighted with a thick green line. This UL and the associated coupling product, as explicitly written out on each plot, is the 95\% exclusion UL for the corresponding ALP mass. This analysis is done for a range of ALP masses and the limits thus obtained from the five magnetars in Fig. 2(1E 2259+586, 4U 0142+61, 1E 1048.1-5937, 1RXS J170849.0-400910 and 1E 1841-045) are shown in Fig. 3.

\section{RESULTS}

From the procedure of spectral analysis in previous section, we present the $95 \%$ CL ULs on $G_{a n} \times g_{a \gamma \gamma}$ with $T_{c}=5 \times 10^{8} \mathrm{~K}$ for the five magnetars in Fig. 3 . For $m_{a} \lesssim 10^{-4} \mathrm{eV}$, the ULs are flat when varying $m_{a}$ as the spectra remain roughly unchanged. The constraints become weak and taper off for $m_{a} \gtrsim 10^{-4} \mathrm{eV}$. This is because the ALP-photon mixing angle becomes small for large ALP masses and the probability of conversion becomes highly reduced. Most of the results shown in this figure can be summarized by the UL at the region when the curves are flat, and we present the 95\% CL ULs on $G_{a n} \times g_{a \gamma \gamma}$ for the magnetars in Table II, for $m_{a}=10^{-7}$ $\mathrm{eV}$.

To see how this result changes when using a different core temperature $T_{c}$, we show in Fig. 4 the $95 \% \mathrm{CL} \mathrm{UL}$ on $G_{a n} \times g_{a \gamma \gamma}$ as a function of $T_{c}$, with the ALP mass fixed at $10^{-7} \mathrm{eV}$. As $T_{c}$ is increased, the ALP production from the core increases appreciably and to saturate the UL on the luminosity, the product of couplings $G_{a n} \times g_{a \gamma \gamma}$ must show a corresponding decrease and leads to a more stringent constraint 3

\footnotetext{
${ }^{3}$ See also [56] for the constraint from diffuse supernova flux.
} 


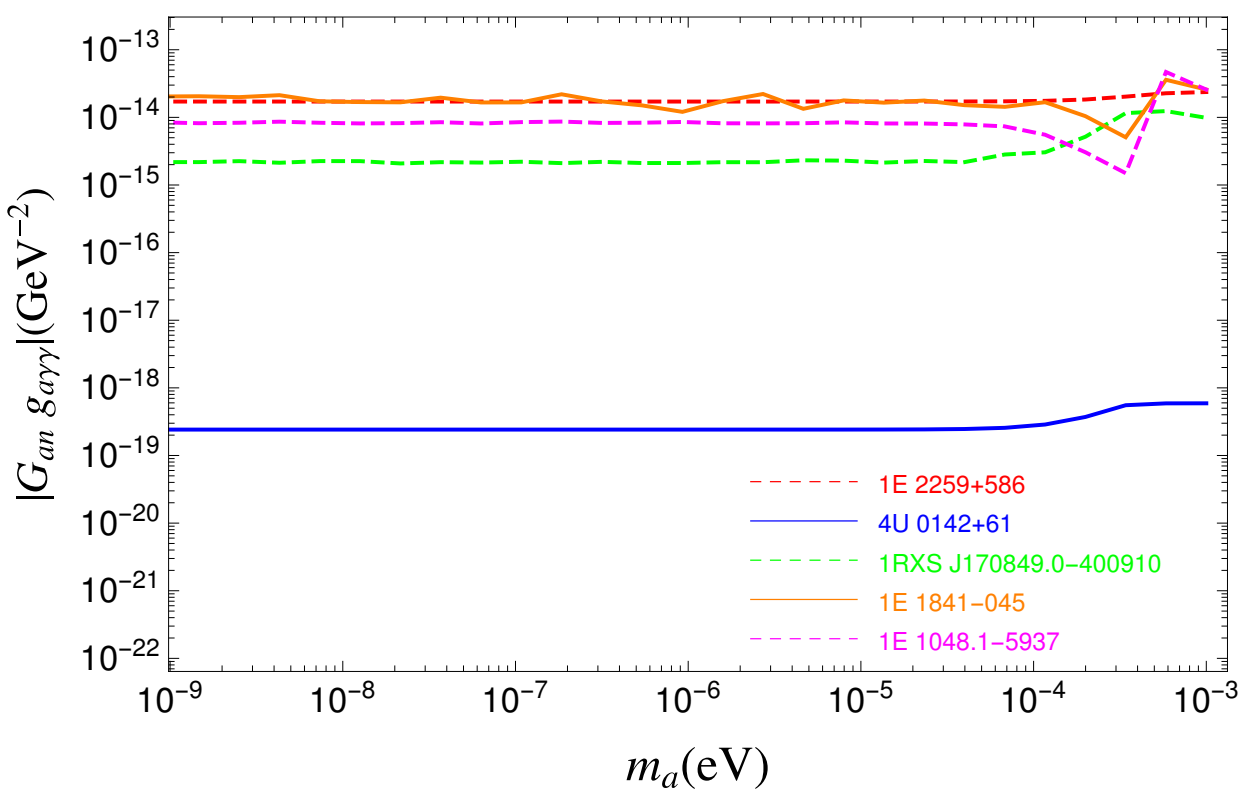

FIG. 3. The 95\% CL upper limits on the coupling $G_{a n} \times g_{a \gamma \gamma}$ for our sample of 5 magnetars, obtained for emissions falling within experimental exclusion bins which overlap with the range $300 \mathrm{keV}-1 \mathrm{MeV}$ assuming $T_{c}=5 \times 10^{8} \mathrm{~K}$.

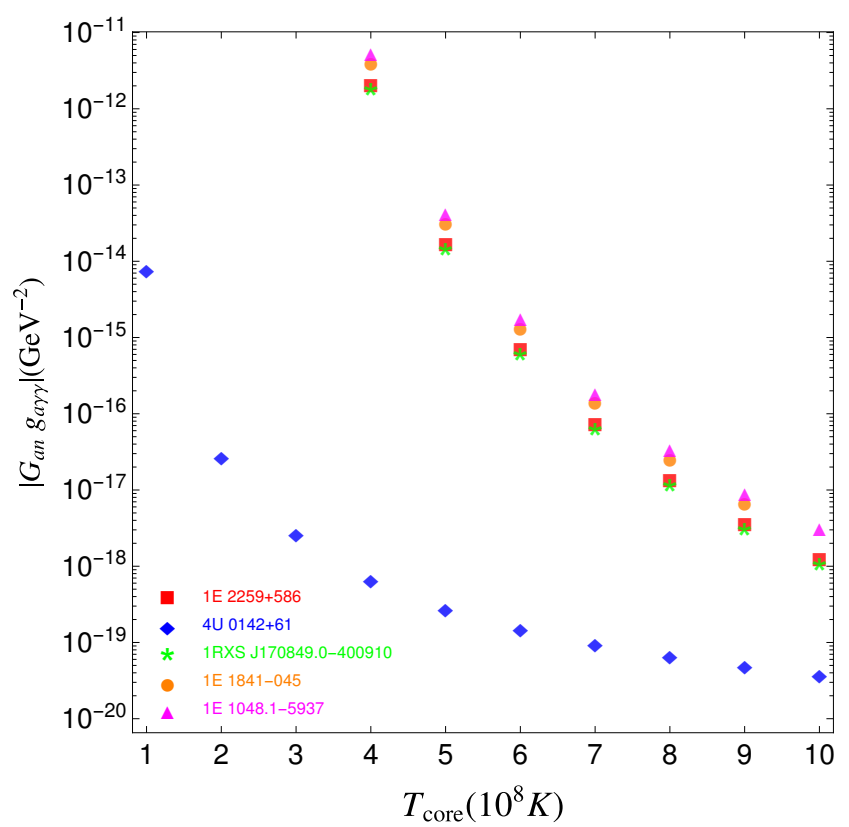

FIG. 4. The $95 \%$ CL UL on the coupling product $G_{a n} \times$ $g_{a \gamma \gamma}$ as $T_{c}$ is varied, for the magnetars in our study. The ALP mass is fixed at $10^{-7} \mathrm{eV}$ and the luminosity from ALPphoton conversion is assumed to saturate UL luminosity listed in Table I

\begin{tabular}{ccc}
\hline Magnetar & $\begin{array}{c}\text { UL Luminosity } \\
\text { at 300-500 keV } \\
10^{35} \mathrm{erg} \mathrm{s}^{-1}\end{array}$ \\
\hline 1E 2259+586 & 6.9 & $1.24 \times 10^{-18}$ \\
4U 0142+61 & 8.8 & $1.17 \times 10^{-18}$ \\
1RXS J170849.0-400910 & 9.8 & $1.06 \times 10^{-18}$ \\
1E 1841-045 & 49.0 & $2.16 \times 10^{-18}$ \\
1E 1048.1-5937 & 55.0 & $2.41 \times 10^{-18}$ \\
\hline
\end{tabular}

TABLE III. Predicted $3 \sigma$ UL on $G_{a n} g_{a \gamma \gamma}\left(\mathrm{GeV}^{-2}\right)$ for our original magnetar sample for future GBM observations at $m_{a}=10^{-7} \mathrm{eV}$.

\section{DISCUSSION: PROPOSED MAGNETAR OBSERVATIONS WITH THE GBM}

The GBM is a non-imaging instrument with a wide field of view. However, it is possible to assign detected events to individual pulsars using the Earth Occultation Technique (EOT) or pulsar timing models. EOT uses a catalogue of sources which exhibit step like changes in photon count rate as seen by the GBM, when the sources are eclipsed by or rise above the Earth limb. In 3 years, EOT has detected 9 of 209 sources between 100-300 keV 67.

The orbital precession of Fermi can be used to apply EOT without a predefined source catalogue. By imaging with a differential filter using the Earth occultation method (IDEOM), the Earth limb is projected onto the sky and used to determine count rates from 600,000 virtual sources with a $0.25^{\circ}$ spacing 68 , identifying 17 new sources. 


\begin{tabular}{cccccc}
\hline Magnetar & $\begin{array}{c}\text { Surface } B \\
\text { Field } \\
10^{14} \mathrm{G}\end{array}$ & $\begin{array}{c}\text { Age } \\
\mathrm{kyr}\end{array}$ & $\begin{array}{c}\text { Distance } \\
\mathrm{kpc}\end{array}$ & $\begin{array}{c}\text { UL Luminosity } \\
\text { at } 300-500 \mathrm{keV} \\
10^{35} \mathrm{erg} \mathrm{s}^{-1}\end{array}$ & $\begin{array}{c}G_{a n} g_{a \gamma \gamma} \\
\left(\mathrm{GeV}^{-2}\right)\end{array}$ \\
\hline SGR 1806-20 & 19.6 & 0.2 & $8.7[57$ & 51.4 & $1.84 \times 10^{-18}$ \\
1E 1547.0-5408 & 3.18 & 0.7 & $4.5[58]$ & 13.7 & $1.29 \times 10^{-18}$ \\
SGR 1900+14 & 7 & 0.9 & $12.5[\underline{[59}$ & 106.0 & $3.13 \times 10^{-18}$ \\
CXOU J171405.7-381031 & 5.01 & 0.9 & $13.2[60]$ & 118.2 & $3.53 \times 10^{-18}$ \\
SGR 1627-41 & 2.25 & 2.2 & $11.0[61]$ & 82.1 & $3.39 \times 10^{-18}$ \\
PSR J1622-4950 & 2.74 & 4.0 & $9.0[62]$ & 55.0 & $2.65 \times 10^{-18}$ \\
SGR J1745-2900 & 2.31 & 4.3 & $8.3[63$ & 46.7 & $2.51 \times 10^{-18}$ \\
Swift J1834.9-0846 & 1.42 & 4.9 & $4.2[64]$ & 12.0 & $1.39 \times 10^{-18}$ \\
XTE J1810-197 & 2.1 & 11.3 & $3.5[65]$ & 8.3 & $1.09 \times 10^{-18}$ \\
SGR 0501+4516 & 1.87 & 15.4 & $2.0[66]$ & 2.7 & $6.35 \times 10^{-19}$ \\
\hline
\end{tabular}

TABLE IV. Predicted $3 \sigma$ UL $G_{a n} g_{a \gamma \gamma}\left(\mathrm{GeV}^{-2}\right)$ values obtainable for proposed future observations of a wider magnetar sample, at $m_{a}=10^{-7} \mathrm{eV}$, assuming a GBM UL energy flux at $300-500 \mathrm{keV}$ of $3.5 \times 10^{-4} \mathrm{MeV} \mathrm{cm}^{-2} \mathrm{~s}^{-1}$ yielding the UL luminosities shown. Distances are from the references given, surface $B$ field and age are from the online ${ }^{27}$ version of the McGill magnetar catalog [20].

The Fermi-GBM Occultation project now monitors 248 sources in the energy range $8 \mathrm{keV}-1 \mathrm{MeV}$ with the majority of the signal seen between $12-50 \mathrm{keV} 4$.

In contrast, the author of [69] uses a pulsar timing method instead. The GBM CTIME data is used to provide photon counts for 4 magnetars, 1RXS J170849.0400910, 1E 1841-045, 4U 0142+61 and 1E 1547.0-5408. The photon counts are attributed to the peak pulsed emission of each magnetar by epoch folding and using timing models (obtained with the Rossi X-ray Timing Explorer), which tags each event by pulsar phase. This count rate is converted to an energy flux for 7 energy channels between $11 \mathrm{keV}-2 \mathrm{MeV}$ by determining the GBM effective area as a function of photon direction, energy and probability of detection of a photon with a given energy. This yields pulsed ULs, just above those obtained by COMPTEL for J170849.0-400910, 1E 1841045 and $4 \mathrm{U} 0142+61$.

The GBM is thus a very useful instrument to determine the UL soft-gamma-ray fluxes of the 23 confirmed magnetars ${ }^{5}$ in the McGill Magnetar Catalog [20, most of which have no ULs defined in the $300 \mathrm{keV}-1 \mathrm{MeV}$ band of interest. We project the possible UL values of $G_{a n} \times g_{a \gamma \gamma}$ that can potentially be obtained using our original sample of magnetars, as well as a wider magnetar sample in Table III and IV]respectively. We use a $3 \sigma$ UL flux sensitivity of $118 \mathrm{mCrab}$ (equivalent to $3.5 \times 10^{-4}$ $\mathrm{MeV} \mathrm{cm}{ }^{-2} \mathrm{~s}^{-1}$ assuming the Crab spectrum in [70]), between 300-500 keV determined from $3 \times$ the error of 3 yr of GBM EOT observations of 4 sources including the Crab 67.

\footnotetext{
4 https://gammaray.msfc.nasa.gov/gbm/science/earth_occ. html accessed on 25th November 2019

J http://www.physics.mcgill.ca/ pulsar/magnetar/main.html accessed on 25th November 2019
}

\section{CONCLUSIONS}

In this paper, we have explored constraints on the product of the ALP-nucleon and ALP-photon couplings. The constraints are obtained from the conversion of ALPs produced in the core of magnetars into photons in the magnetosphere. When interpreting our results in Figure 3 , the following caveats apply: since the magnetars in our selection have no published values of $T_{c}$, the results are displayed for a benchmark $T_{c}$ of $5 \times 10^{8} \mathrm{~K}$. We further show the limits that can be obtained by varying $T_{c}$ in Figure 4 for a fixed ALP mass of $10^{-7} \mathrm{eV}$. We also note that a more stringent limit can be obtained by a combined analysis of the upper limits from all magnetars.

Our results motivate a program of studying quiescent soft-gamma-ray emission from magnetars in the 300 keV-1 MeV band with Fermi-GBM. The GBM will be able to determine the UL soft-gamma-ray fluxes of confirmed magnetars, most of which have no ULs defined in soft gamma-rays. With these ULs, it is possible that even more stringent constraints on the product of the ALP couplings may be obtained.

\section{ACKNOWLEDGEMENTS}

HG and KS are supported by DOE Grant DESC0009956. KS would like to thank Jean-Francois Fortin for many discussions on ALP-photon conversions near magnetars, to be incorporated into a forthcoming publication [71. He would also like to thank KITP Santa Barbara for hospitality during part of the time that this work was completed. AMB and PMC acknowledge the financial support of the UK Science and Technology Facilities Council consolidated grant ST/P000541/1. 
[1] R. D. Peccei and H. R. Quinn, Phys. Rev. Lett. 38, 1440 (1977), [,328(1977)].

[2] S. Weinberg, Phys. Rev. Lett. 40, 223 (1978).

[3] M. Dine, W. Fischler, and M. Srednicki, Phys. Lett. 104B, 199 (1981)

[4] J. Preskill, M. B. Wise, and F. Wilczek, Phys. Lett. B 120, 127 (1983).

[5] L. Abbott and P. Sikivie, Phys. Lett. B 120, 133 (1983).

[6] P. W. Graham, I. G. Irastorza, S. K. Lamoreaux, A. Lindner, and K. A. van Bibber, Ann. Rev. Nucl. Part. Sci. 65, 485 (2015), arXiv:1602.00039 [hep-ex]

[7] J.-F. Fortin and K. Sinha, JHEP 06, 048 (2018), arXiv:1804.01992 [hep-ph].

[8] J.-F. Fortin and K. Sinha, JHEP 01, 163 (2019), arXiv:1807.10773 [hep-ph].

[9] S. J. Lloyd, P. M. Chadwick, and A. M. Brown, Phys. Rev. D100, 063005 (2019), arXiv:1908.03413 [astro-ph.HE]

[10] D. E. Morris, Phys. Rev. D34, 843 (1986).

[11] G. Raffelt and L. Stodolsky, Phys. Rev. D37, 1237 (1988).

[12] N. Iwamoto, Phys. Rev. Lett. 53, 1198 (1984)

[13] R. P. Brinkmann and M. S. Turner, Phys. Rev. D38, 2338 (1988).

[14] M. Buschmann, R. T. Co, C. Dessert, and B. R. Safdi, "X-ray search for axions from nearby isolated neutron stars," (2019), arXiv:1910.04164 [hep-ph].

[15] R. Kothes and T. Foster, Astrophys. J. 746, L4 (2012).

[16] L. Kuiper, W. Hermsen, P. R. den Hartog, and W. Collmar, Astrophys. J. 645, 556 (2006), arXiv:astro-ph/0603467 [astro-ph]

[17] M. Durant and M. H. van Kerkwijk, Astrophys. J. 650, 1070 (2006), arXiv:astro-ph/0606027 [astro-ph].

[18] P. R. den Hartog, L. Kuiper, W. Hermsen, V. M. Kaspi, R. Dib, J. Knoedlseder, and F. P. Gavriil, Astron. Astrophys. 489, 245 (2008), arXiv:0804.1640 [astro-ph]

[19] W. Tian and D. A. Leahy, Astrophys. J. 677, 292 (2008), arXiv:0709.4667 [astro-ph].

[20] S. A. Olausen and V. M. Kaspi, Astrophys. J. Suppl. 212, 6 (2014), arXiv:1309.4167 [astro-ph.HE],

[21] R. Perna, W. C. Ho, L. Verde, M. van Adelsberg, and R. Jimenez, Astrophys. J. 748, 116 (2012), arXiv:1201.5390 [astro-ph.HE]

[22] F. V. Day, Phys. Lett. B 753, 600 (2016), arXiv:1506.05334 [hep-ph]

[23] M. Fairbairn, Phys. Rev. D 89, 064020 (2014), arXiv:1310.4464 [astro-ph.CO].

[24] O. Benhar, "From yukawa's theory to the one-pion-exchange potential," (2017).

[25] P. Carenza, T. Fischer, M. Giannotti, G. Guo, G. Martínez-Pinedo, and A. Mirizzi, JCAP 10, 016 (2019), arXiv:1906.11844 [hep-ph].

[26] J.-F. Fortin and K. Sinha, JHEP 06, 048 (2018), arXiv:1804.01992 [hep-ph].

[27] S. P. Harris, J.-F. Fortin, K. Sinha, and M. G. Alford, (2020), arXiv:2003.09768 [hep-ph]

[28] B. Liu, V. Greco, V. Baran, M. Colonna, and M. Di Toro, Phys. Rev. C 65, 045201 (2002), arXiv:nucl-th/0112034.

[29] W.-j. Fu, G.-h. Wang, and Y.-x. Liu, Astrophys. J. 678, 1517 (2008)

[30] W. Dittrich and H. Gies, Springer Tracts Mod. Phys. 166, 1 (2000).

[31] A. E. Shabad, Annals Phys. 90, 166 (1975).

[32] W.-y. Tsai, Phys. Rev. D10, 2699 (1974).

[33] D. B. Melrose and R. J. Stoneham, Nuovo Cim. A32, 435 (1976)

[34] L. F. Urrutia, Phys. Rev. D17, 1977 (1978)

[35] F. Karbstein, Proceedings, International Wokshop on Strong Field Problems in Quantum Theory: Tomsk, Russia, June 6-11, 2016, Russ. Phys. J. 59, 1761 (2017), arXiv:1607.01546 [hep-ph].

[36] F. Karbstein and R. Shaisultanov, Phys. Rev. D91, 085027 (2015), arXiv:1503.00532 [hep-ph]

[37] F. Karbstein, Phys. Rev. D88, 085033 (2013), arXiv:1308.6184 [hep-th],

[38] J. S. Heyl and L. Hernquist, J. Phys. A30, 6485 (1997), arXiv:hep-ph/9705367 [hep-ph],

[39] L. Kuiper, W. Hermsen, and M. Mendez, Astrophys. J. 613, 1173 (2004), arXiv:astro-ph/0404582 [astro-ph].

[40] D. Gotz, S. Mereghetti, A. Tiengo, and P. Esposito, Astron. Astrophys. 449, L31 (2006), arXiv:astro-ph/0602359 [astro$\mathrm{ph}]$

[41] P. R. den Hartog, L. Kuiper, and W. Hermsen, Astron. Astrophys. 489, 263 (2008), arXiv:0804.1641 [astro-ph],

[42] J. Li, N. Rea, D. F. Torres, and E. de Ona-Wilhelmi, Astrophys. J. 835, 30 (2017), arXiv:1607.03778 [astro-ph.HE].

[43] M. G. Baring and A. K. Harding, Conference on Isolated Neutron Stars: From the Interior to the Surface London, England, April 24-28, 2006, Astrophys. Space Sci. 308, 109 (2007), arXiv:astro-ph/0610382 [astro-ph].

[44] S. Zane, R. Turolla, L. Nobili, and N. Rea, Adv. Space Res. 47, 1298 (2011), arXiv:1008.1537 [astro-ph.HE]

[45] A. M. Beloborodov, Astrophys. J. 762, 13 (2013) arXiv:1201.0664 [astro-ph.HE]

[46] Z. Wadiasingh, M. G. Baring, P. L. Gonthier, and A. K. Harding, Astrophys. J. 854, 98 (2018), arXiv:1712.09643 [astroph.HE],

[47] K. Hu, M. G. Baring, Z. Wadiasingh, and A. K. Harding, Mon. Not. Roy. Astron. Soc. 486, 3327 (2019), arXiv:1904.03315 [astro-ph.HE]

[48] A. C. Collazzi et al., Astrophys. J. Suppl. 218, 11 (2015), arXiv:1503.04152 [astro-ph.HE]

[49] T. Enoto, S. Kisaka, and S. Shibata, Rept. Prog. Phys. 82, 106901 (2019).

[50] V. M. Kaspi and A. Beloborodov, Ann. Rev. Astron. Astrophys. 55, 261 (2017), arXiv:1703.00068 [astro-ph.HE]. 
[51] A. D. Kaminker, D. G. Yakovlev, A. Y. Potekhin, N. Shibazaki, P. S. Shternin, and O. Y. Gnedin, Mon. Not. Roy. Astron. Soc. 371, 477 (2006), arXiv:astro-ph/0605449 [astro-ph].

[52] S. Dall'Osso, S. N. Shore, and L. Stella, Mon. Not. Roy. Astron. Soc. 398, 1869 (2009), arXiv:0811.4311 [astro-ph],

[53] W. C. G. Ho, K. Glampedakis, and N. Andersson, Mon. Not. Roy. Astron. Soc. 422, 2632 (2012), arXiv:1112.1415 [astro-ph.HE]

[54] A. Y. Potekhin, G. Chabrier, and D. G. Yakovlev, Conference on Isolated Neutron Stars: From the Interior to the Surface London, England, April 24-28, 2006, Astrophys. Space Sci. 308, 353 (2007), arXiv:astro-ph/0611014 [astro-ph].

[55] A. M. Beloborodov and X. Li, Astrophys. J. 833, 261 (2016), arXiv:1605.09077 [astro-ph.HE].

[56] F. Calore, P. Carenza, M. Giannotti, J. Jaeckel, and A. Mirizzi, Phys. Rev. D 102, 123005 (2020), arXiv:2008.11741 [hep-ph]

[57] J. L. Bibby, P. A. Crowther, J. P. Furness, and J. S. Clark, MNRAS 386, L23 (2008), arXiv:0802.0815 [astro-ph].

[58] A. Tiengo, G. Vianello, P. Esposito, S. Mereghetti, A. Giuliani, E. Costantini, G. L. Israel, L. Stella, R. Turolla, S. Zane, N. Rea, D. Götz, F. Bernardini, A. Moretti, P. Romano, M. Ehle, and N. Gehrels, ApJ 710, 227 (2010), arXiv:0911.3064 [astro-ph.HE]

[59] B. Davies, D. F. Figer, R.-P. Kudritzki, C. Trombley, C. Kouveliotou, and S. Wachter, ApJ 707, 844 (2009), arXiv:0910.4859 [astro-ph.SR]

[60] W. W. Tian and D. A. Leahy, MNRAS 421, 2593 (2012), arXiv:1201.0731 [astro-ph.GA].

[61] S. Corbel, C. Chapuis, T. M. Dame, and P. Durouchoux, ApJ 526, L29 (1999), arXiv:astro-ph/9909334 [astro-ph]

[62] L. Levin, M. Bailes, S. Bates, N. D. R. Bhat, M. Burgay, S. Burke-Spolaor, N. D'Amico, S. Johnston, M. Keith, M. Kramer, S. Milia, A. Possenti, N. Rea, B. Stappers, and W. van Straten, ApJ 721, L33 (2010), arXiv:1007.1052 [astro-ph.HE]

[63] G. C. Bower, A. Deller, P. Demorest, A. Brunthaler, R. Eatough, H. Falcke, M. Kramer, K. J. Lee, and L. Spitler, ApJ 780, L2 (2014) arXiv:1309.4672 [astro-ph.GA]

[64] D. A. Leahy and W. W. Tian, AJ 135, 167 (2008), arXiv:0708.3377 [astro-ph],

[65] A. H. Minter, F. Camilo, S. M. Ransom, J. P. Halpern, and N. Zimmerman, ApJ 676, 1189 (2008), arXiv:0705.4403 [astro-ph].

[66] L. Lin, C. Kouveliotou, M. G. Baring, A. J. van der Horst, S. Guiriec, P. M. Woods, E. Göğüș, Y. Kaneko, J. Scargle, J. Granot, R. Preece, A. von Kienlin, V. Chaplin, A. L. Watts, R. A. M. J. Wijers, S. N. Zhang, N. Bhat, M. H. Finger, N. Gehrels, A. Harding, L. Kaper, V. Kaspi, J. Mcenery, C. A. Meegan, W. S. Paciesas, A. Pe'er, E. Ramirez-Ruiz, M. van der Klis, S. Wachter, and C. Wilson-Hodge, ApJ 739, 87 (2011)

[67] C. A. Wilson-Hodge et al., Astrophys. J. Suppl. 201, 33 (2012) arXiv:1201.3585 [astro-ph.HE]

[68] J. Rodi, M. L. Cherry, G. L. Case, A. Camero-Arranz, V. Chaplin, M. H. Finger, P. Jenke, and C. A. Wilson-Hodge, Astron. Astrophys. 562, A7 (2014), arXiv:1304.1478 [astro-ph.HE]

[69] F. ter Beek, FERMI GBM detections of four AXPs at soft gamma-rays, Thesis (2012).

[70] Kuiper, L., Hermsen, W., Cusumano, G., Diehl, R., Schönfelder, V., Strong, A., Bennett, K., and McConnell, M. L., A\&A 378, 918 (2001)

[71] J.-F. Fortin, H. Guo, and K. Sinha, Work in progress. 